\title{
POINT CONTACT STUDIES OF HIGH $T_{\mathbf{c}}$ SUPERCONDUCTORS
}

\author{
B. Susta
}

Institute of Physics, Poznań Technical University, Piotrowo 3, 60-965 Poznań, Poland

\author{
S.C. Meepagala and E.L. Wolf
}

Department of Physics, Polytechnic University, Brooklyn, New York 11201, USA

(Received December 14, 1992; in final form May 24, 1993)

\begin{abstract}
Point contact spectroscopy experiments were performed on both single crystals $\mathrm{Bi}_{2} \mathrm{Sr}_{2} \mathrm{CaCu}_{2} \mathrm{O}_{8+y}$ and $\mathrm{BiSrCaCuO} / \mathrm{Ag}$ sintered material using gold tips. The spectra exhibit tunneling structure, which are interpreted in terms of the energy gap structure $2 \Delta=58 \mathrm{meV}$ at $4.2 \mathrm{~K}$. We observed the strong Andreev reflection of the $\mathrm{Au}-\mathrm{BiSrCaCuO} / \mathrm{Ag}$ boundary.
\end{abstract}

PACS numbers: 74.50.+r, 74.70. Tx

\section{Introduction}

Point contact spectroscopy (PCS) is an useful alternative to tunneling spectroscopy, in obtaining properties of superconductors $[1,2]$. This technique is especially suited for studying new high $T_{\mathrm{c}}$ superconductors, as the preparation of conventional tunnel junctions seems difficult. It has been previously shown that good quality metallic point contacts can be established even when the surface has some insulating oxides [3]. Thus, point contact studies are a logical step in the study of high $T_{\mathrm{c}}$ materials. Some results which were obtained using this technique can be found in the literature $[4,5]$. Experimental method provides an information about the electronic structure, coupling mechanism of superconductors, the size of the encrgy gap, phonon density of state, and magnitude of the electron-phonon coupling.

Other obvious benefits from point contact studies of high $T_{\mathrm{c}}$ superconductors are:

i) We can get an understanding of the proximity effect of high $T_{\mathrm{c}}$ materials. This is important since superconductor-normal metal-superconductor (SNS) structures with Josephson characteristics can be made basing on the proximity effect. 
ii) We can learn about the nature of metallic contacts to the high $T_{\mathfrak{c}}$ materials. This is important in applications of new oxide materials (e.g. in devices, magnets etc.).

iii) We can study the excitation spectra of high $T_{\mathrm{c}}$ materials. Since it is possible for the point to penetrate the surface layer and make contact with good superconducting boundary, we can expect to avoid the artifacts due to poor quality of the surface layer.

In this paper, we show data obtained from point contact spectroscopy experiments made on single crystals $\mathrm{Bi}_{2} \mathrm{Sr}_{2} \mathrm{CaCu}_{2} \mathrm{O}_{8+y}$ (BSCCO) and sintered material $\mathrm{BiSrCaCuO} / \mathrm{Ag}$. The strong Andreev reflection of the $\mathrm{Au}-\mathrm{BiSrCaCuO} / \mathrm{Ag}$ boundary was observed. In our experiments on the single crystals we also observed energy gap dependence versus temperature [10]. The results for $4.2 \mathrm{~K}$ are compared with $\Delta$ for sandwich junction on similar crystals.

\section{Experimental method}

The simple point contact apparatus shown in Fig. 1 was used for this preliminary investigation. We mounted the sample facing downward, using GE varnish. The screw had a thin gold wire of diameter $0.3 \mathrm{~mm}$ attached to it. We cut the gold wire at an angle of approximately $45^{\circ}$ to define a sharp point. The point contact

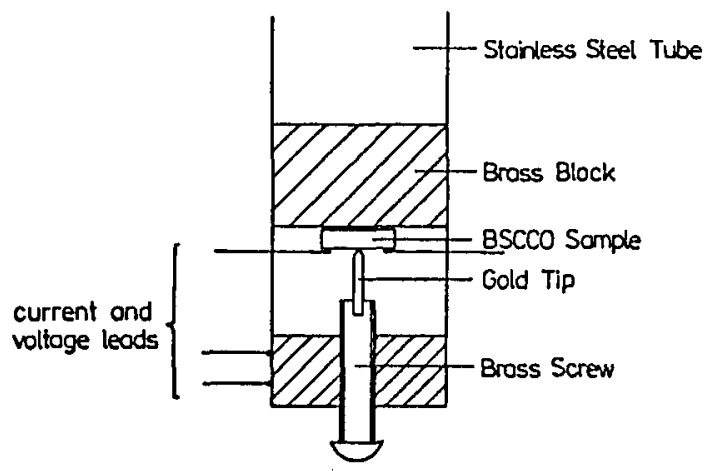

Fig. 1. Schematic diagram of point contact sample holder.

was established by rotating the screw, and then we immersed the sample holder in liquid helium. Electrical contact to the sample was made by attaching two leads using silver paint. Electrical contact to the tip was made through the screw. Using conventional apparatus, we recorded $I-V$ and $\mathrm{d} V / \mathrm{d} I-V$ curves on a $x-y$ recorder. During the $\mathrm{d} V / \mathrm{d} I$ measurements, the voltage modulation across the sample was kept below $0.5 \mathrm{mV}$ and we used low-frequency modulation [6].

\section{Results and discussion}

An interesting $I-V$ and $\mathrm{d} V / \mathrm{d} I-V$ curves which were obtained for a sintered high $T_{\mathrm{c}}$ sample are shown in Fig. 2. 


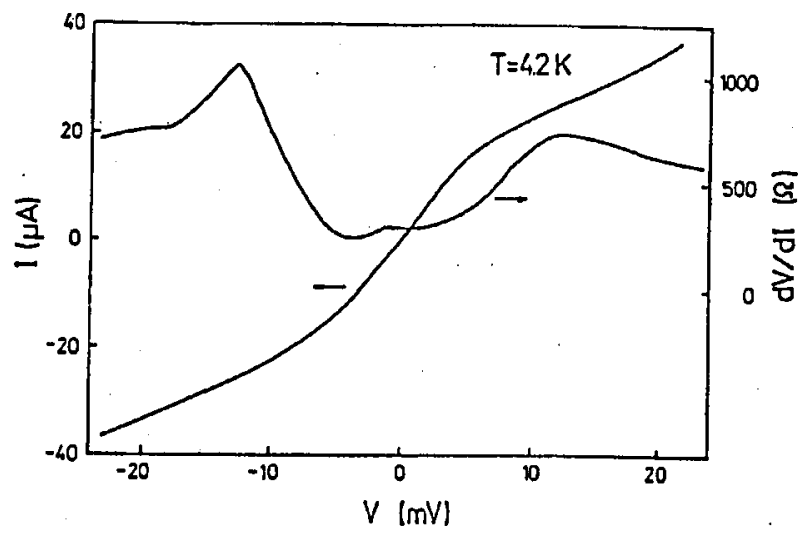

Fig. 2. Typical spectra $I-V$ and differential resistance $\mathrm{d} V / \mathrm{d} I$ vs. dc bias voltage at $4.2 \mathrm{~K}$.

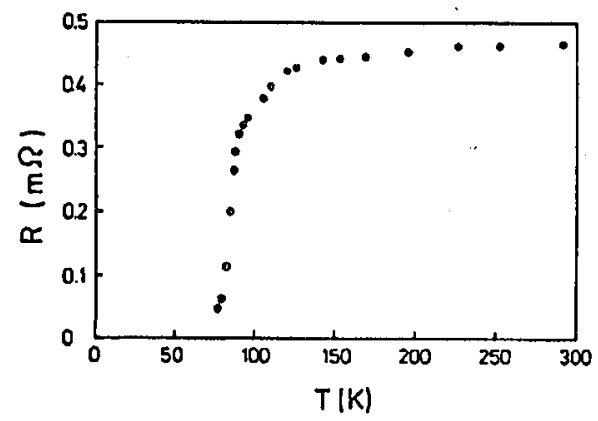

Fig. 3. Resistance curve for $83.3 \% \mathrm{Bi}$ compound $+16.7 \% \mathrm{Ag}$ as a function of temperature.

This sample also had $16.7 \% \mathrm{Ag}$ in it and the $T_{\mathrm{c}}$ (for zero resistance) was $75 \mathrm{~K}$. A resistance curve for this sample is shown in Fig. 3. Judging from this curve and also from X-ray analysis (not shown data), our sample consisted almost of single phase.

The differential resistance (Fig. 2) for small bias voltage $(<5 \mathrm{mV})$ was smaller than that for higher bias voltages $(>10 \mathrm{mV})$. The ratio of the resistances in these two regimes was 2 on the positive bias side and somewhat larger on the negative bias side. We believe that this reduction of the resistance at low bias voltages is a manifestation of the Andreev reflection at the normal-superconducting boundary [7]. To see a large effect as seen in Fig. 2, the point must have made a nearly ideal contact (that is, no impurity scattering at the boundary) with a superconducting area.

Tunneling characteristics which were obtained for a single crystal BSCCO sample ( $a-b$ plane) are shown in Fig. 4. No indication of the Andreev reflection is seen, and the $I-V$ curve looks like those typically reported in the tunneling 


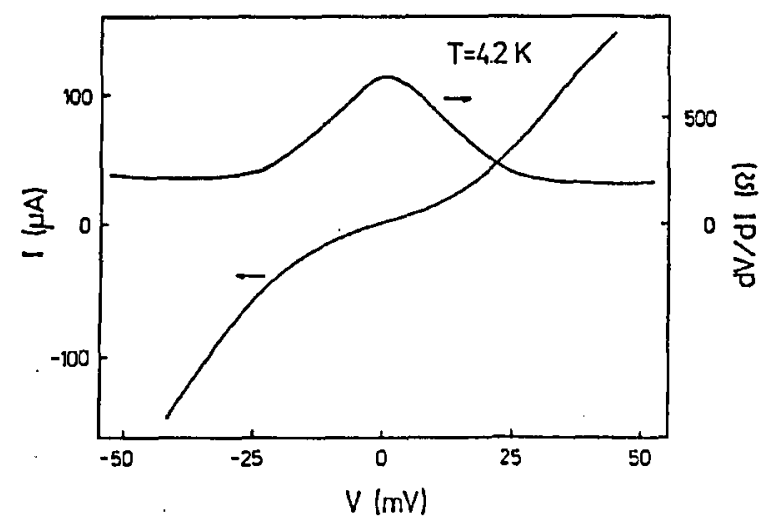

Fig. 4. Tunneling spectra obtained from BSCCO single crystals at $4.2 \mathrm{~K}$.

experiments. We attribute the lack of the Andreev reflection to the non-metallic $\mathrm{BiO}$ layer which exists on the surface of the BSCCO sample [8]. Such a barrier can prevent metallic-like point contact and reduces the probability of the occurrence of the Andreev reflection [9]. In the sintered material, the crystallites are randomly oriented and the Au-tip has a chance of making contact with a suitable facet. No sharp gap features are seen, in contrast to clear gap structures in the planar tunnel junction for the same single crystal [10]. Tunneling charactcristic yielded $2 \Delta=58 \mathrm{meV}$ at the $4.2 \mathrm{~K}$. The differential resistance $\mathrm{d} V / \mathrm{d} I$ versus voltage $V$ of a point contact between superconductor and normal metal, should he responsible to explain the gap structure of high $T_{\mathrm{c}}$ superconductors. These data are in good agreement with experimental data by $\mathrm{M}$. Lee et al. [11] using evaporated Nb film on similar 2212 crystals.

Figure 5 shows the measurements done on the same junction which was used for the data in Fig. 4, but after we had applied a high bias voltage (1 V). During the measurements using bias voltages exceeding $1 \mathrm{~V}$, we saw hysteretic behaviors which we attributed to the heating at the point of contact. This process irreversibly changed the junction properties as evidenced in Fig. 5. Perhaps we damaged the $\mathrm{BiO}$ layer or made some alloying with tip matcrial. The minimum in $\mathrm{d} V / \mathrm{d} I$ at zero bias, seen in Fig. 5, is probably due to the onset of the Andreev reflection at the new boundary.

Apart from the above data (Figs. 2, 4, 5) we observed various other structures possibly due to the excitations around the Fermi energy, Coulomb blockade, etc. For clarity, we are not showing these data in this paper.

In summary, we report point contact spectroscopy experiments on the high temperature superconductors. We observed an indication of strong Andreev reflection at the $\mathrm{Au}-\mathrm{BiSrCaCuO} / \mathrm{Ag}$ boundary. This shows that good metallic point contacts can be made to sintered $\mathrm{BiSrCaCuO} / \mathrm{Ag}$ material.

The determination of the energy gap by Andreev reflection experiments is complicated by other energy dependent processes, like electron-phonon scattering. On the $a-b$ plane of the single crystals $\mathrm{Bi}_{2} \mathrm{Sr}_{2} \mathrm{CaCu}_{2} \mathrm{O}_{8+y}$, we failed to get as good 


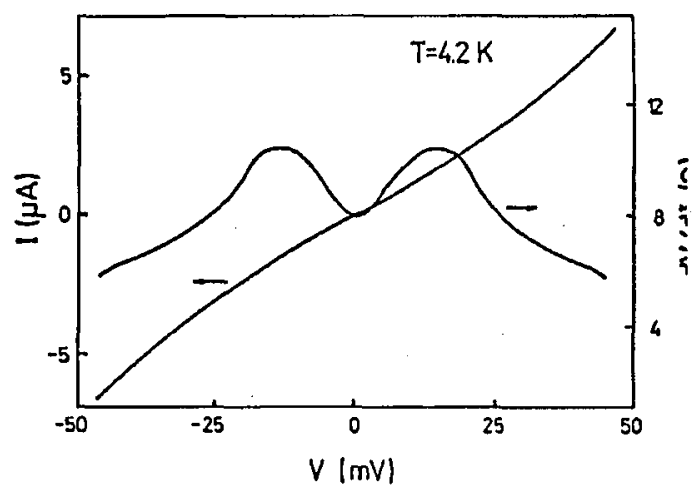

Fig. 5. The $I-V$ and $\mathrm{d} V / \mathrm{d} I$ vs. $V$ curves for a BSCCO single crystals with Au tip of point contact at $4.2 \mathrm{~K}$ after applying a high bias voltage (1 V).

point contacts as for sintered high $T_{\mathrm{c}}$ sample. We attribute this to the non-metallic $\mathrm{BiO}$ layer which resides on $\mathrm{BSCCO}$ single crystal.

Bias voltage (current) should be kept low throughout the whole experiment in order not to disturb the propertics of $\Lambda \mathrm{u}$-high temperature superconductors boundary.

\section{Acknowledgment}

This work has been partially supported by the Committee for Scientific Research through grant No. 2600/2/01.

\section{References}

[1] A.G.M. Jansen, F.M. Mueller, P. Wyder, Science 190, 1037 (1978).

[2] A.G.M. Jansen, A.P. van Gelder, P. Wyder, J. Phys. C 13, 6073 (1980).

[3] G.E. Blonder, M. Tinkham, Phys. Rev. B 27, 112 (1983).

[4] M.D. Kirk, D.P.E. Smith, D.B. Mitzi, J.Z. Sun, D.J. Webb, K. Char, M.R. Hahn, M. Naito, B. Oh, M.R. Beasley, T.II. Geballe, R.II. IIammond, A. Kapitulnik, C.F. Quate, Phys. Rev. B 35, 8850 (1987).

[5] P.J.M. van Bentum, II.F.C. Iloevers, II. van Kempen, L.E.C. van de Leemput, M.J.M.F. de Nivelle, L.W.M. Schreurs, R.T.M. Smokers, P.A.A. Teunissen, Physica C 153-155, 1718 (1988).

[6] E.L. Wolf, Principles of Tunneling Spectroscopy, Oxford University Press, New York 1985, p. 474.

[7] A.F. Andreev, Sov. Phys.-JETP 19, 1228 (1964).

[8] C.K. Shih, R.M. Feenstra, J.R. Kirtlcy, G.V. Cltandrasekhar, Phys. Rev. B 40, 2682 (1989).

[9] G.E. Blonder, M. Tinkham, T.M. Klapwijk, Phys. Rev. B 25, 4515 (1982).

[10] B. Susła, H.J. Tao, E.L. Wolf, Ferroelectrics 128, 225 (1992).

[11] M. Lee, D.B. Mitzi, A. Kapitulnik, M.R. Beasley, Phys. Rev. B 39, 1008 (1989). 